\title{
MINIMUM EMISSION UNCONVENTIONAL MAGLEV ASSISTED TAKE-OFF
}

\author{
Andrzej Majka \\ Rzeszow University of Technology \\ Department of Aircrafts and Aircraft Engines \\ Powstancow Warszawy Street 12, 35-959 Rzeszow, Poland \\ tel.:+48 178651604 , fax: +48178651942 \\ e-mail: andrzej.majka@prz.edu.pl
}

\begin{abstract}
Among the most important problems faced by the air transport today there can be mentioned some negative influences of aircraft and airports on the environment and the increasing costs of air transport. One of the possibilities to improve the situation is to work out innovative solutions aimed at decreasing of the aircraft pollution and improving the transport effectiveness. Among the most innovative ideas is the use of magnetic levitation $(M A G L E V)$ to aid the take-off and landing of the transport aircraft. The "Integrated Ground and on-Board system for Support of the Aircraft Safe Take-off and Landing" - GABRIEL, deals with radically new integration of the MAGLEV technology into the air transportation system that contains aircraft, airport, air traffic control, authority, logistic and operational support, maintenance, etc. GABRIEL idea includes operation of the aircraft without the conventional undercarriage system and using the ground based power and supporting systems for take-offs and landings. In case of using the GABRIEL system as a catapult, the aircraft on the track must be accelerated to the speed permitting to reach the altitude of $300 \mathrm{~m}$ or more. Such take-off and climb results in minimum noise and minimal emission of substances harmful for the environment. After reaching the altitude of $300 \mathrm{~m}$, the aircraft may follow its flight with using different flight strategies. Different conditions of the unconventional take-off needs to shape other way the trajectory of the initial stage of the aircraft flight after the lift-off. The aim of the present work was to determine the optimal conditions of the lift-off and the optimal trajectory of the initial climb minimizing the emission. Simplified realization of the RitzGalerkin method was used in this work, which uses an approximate solution to boundary value problems for determining the optimal flight trajectory.
\end{abstract}

Keywords: air transport, green trajectory, take-off, aircraft emission, magnetic levitation

\section{Introduction}

Very high air traffic density in the largest airports and in their vicinity involves that the air traffic in the largest airports and their areas of operations approaches the capacity limits. Such high density of the air traffic adversely influences the natural environment in the vicinity of the airports due to the increasing cumulative noise level and the concentration of environmentally hazardous substances. One of the possibilities to improve the situation is to work out innovative solutions aimed at decreasing the aircraft pollution and improving the transport effectiveness. There are several technologies that could be applied to reduce the harmful influence of the air transport on the environment. Novel ideas include for example operation of the aircraft without the conventional undercarriage system and using the ground based power and supporting systems for take-offs and landings. If ground launched technologies that accelerate and "launch" the aircraft into the air are applied, than the power requirements can be substantially reduced even over the initial climb phase, as only such power would be needed that is required to manoeuvre and fly. One of the major concepts is using magnetic levitation (MAGLEV) technology to support aircraft take-off and landing. The idea of use supporting system using MAGLEV technology was described at $[9-11,13,16]$. In case of using the magnetic levitation technology, the airframe weight can be considerably reduced, since the undercarriage system could be lighter or even ignored. The required engine power is determined by the take-off phase in which a substantial thrust is needed. Therefore, if the aircraft could take-off and start the initial climb phase with the ground power, the installed power may be reduced, resulting in less weight, less drag and less 
overall fuel consumption, which leads to emission reduction. Different conditions of the take-off give possibilities to shape the trajectory of the initial stage of the aircraft climb after the take-off in order to decrease the negative influence on the environment. The aim of the present work was to determine the optimal conditions of the take-off and the optimal trajectory of the initial climb of the aircraft aided in the ground phase of the take-off by the system using the MAGLEV technology, minimizing the fuel consumption. The simplified algorithm of optimization of the flight trajectory was used in this work; it uses the method of approximation of the flight path by the third degree polynomial. Optimization of the departure trajectory minimizing fuel consumption or noise emissions can become the basis for working out new procedures for a new kind of takeoff modified in relation to the optimal solution, which will increase safety of this segment of the flight.

\section{Problem formulation}

The climb performance of an aircraft is an important design requirement for establishing trajectories to reach a specified altitude and airspeed after take-off in some optimal manner. Usually for transport aircraft, a climb segment may follow a trajectory designed to achieve optimal fuel consumption or a minimum time. Trajectory optimization problems to minimize aircraft fuel consumption, noise or time of climb had been studied by various contributors $[1,5,7,8,14,15$, 18]. The problem is formulated as the calculus of variations where an objective function is minimized while satisfying initial/final conditions and path constrains for a flying vehicle. Although some techniques such as the dynamic programming of Bellman [4] and the maximum principle of Pontryagin [12] have been developed and used. Simplified realization of the RitzGalerkin method was used in this work, which uses an approximate solution to boundary value problems for determining the optimal flight trajectory described by Taranienko et al. [17]. The method allows determining the optimal trajectory of the flight satisfying the initial/final conditions and control functions and path constrains for an aircraft. The case of optimal climbing and acceleration in the vertical plane with the determined conditions and appropriate constrains was considered. General stating of the task supposes determining the optimal trajectory of movement of a flying vehicle described by the system of ordinary differential equations:

$$
x_{i}=f_{i}\left(x_{1}, \ldots, x_{n}, u_{1}, \ldots, u_{m}\right), \quad i=1,2, \ldots, n, \quad m \leq n,
$$

fulfilling boundary conditions:

$$
\begin{aligned}
& x\left(t_{0}\right)=\left\{x_{1}\left(t_{0}\right), x_{2}\left(t_{0}\right), \ldots, x_{n}\left(t_{0}\right)\right\}, \quad x \in \mathbb{R}^{n}, \\
& x\left(t_{f}\right)=\left\{x_{1}\left(t_{f}\right), x_{2}\left(t_{f}\right), \ldots, x_{n}\left(t_{f}\right)\right\}
\end{aligned}
$$

and constraint for state variables and control variables:

$$
\begin{aligned}
& x_{i \text { min }}(t) \leq x_{i}(t) \leq x_{i \max }(t), \\
& u_{j \text { min }}(t) \leq u_{j}(t) \leq u_{j \max }(t), \quad j=1,2, \ldots, m,
\end{aligned}
$$

where:

$x_{i} \quad-$ state variables,

$u_{j}-$ control variables,

$t_{0}, t_{f}-$ initial and final times.

The technical characteristics of the flying vehicle are known and can be written as:

$$
x_{T}=\left(x_{T 1}, \ldots, x_{T r}\right), \quad x_{r} \in \mathbb{R}^{r} .
$$

The optimal trajectory $x(t), t \in\left(t_{0}, t_{f}\right)$ has to be found which minimizes the functional: 


$$
J(x(t))=\int_{t_{0}}^{t_{f}} f_{0}(X, U) d t,
$$

and the control corresponding to it is:

$$
U(t)=\left(u_{1}(t), \ldots, u_{m}(t)\right), \quad U \in \mathbb{R}^{m}
$$

In the case of the current task the system of differential equations (1) commonly employed in the aircraft trajectory analysis is the following six-dimension system derived at the centre of mass of the aircraft [6]:

$$
\begin{aligned}
& \stackrel{\circ}{V}=g\left(\frac{T \cos \alpha-D}{m g}-\sin \gamma\right), \\
& \stackrel{\circ}{\gamma}=\frac{1}{m V}((T \sin \alpha+L) \cos \varphi-m g \cos \gamma), \\
& \stackrel{\circ}{\psi}=\frac{(T \sin \alpha+L) \sin \varphi}{m V \cos \gamma}, \\
& \circ \\
& \dot{x}=V \cos \gamma \cos \psi, y=V \cos \gamma \sin \psi, z=\stackrel{\circ}{\circ}=V \sin \gamma .
\end{aligned}
$$

Parameters $V, \gamma, \psi, \alpha$ and $\varphi$ are respectively the speed, the angle of descent, the yaw angle, the angle of attack and the roll angle. $(x, y, z=h)$ is the position of the aircraft. The variables $T, D, L, m$ (technical characteristics - 4) and $g$ are respectively the engine thrust, the drag force, the lift force, the aircraft mass and the gravitational acceleration. It was considering a 2-Degree Of Freedom (DOF) dynamic model that describes the point variable-mass motion of the aircraft over a flat Earth model. A standard atmosphere is defined with ISA (International Standard Atmosphere). $C_{L}$ is, in general, a function of the angle of attack and the Mach number, i.e., $C_{L}=C_{L}(\alpha, M)$. The lift coefficient is used as a variable rather than the angle of attack. The aircraft performance model is used from [9] and [11].

\section{Results}

In the frames of this work the optimal trajectory of climbing of the aircraft with the characteristics of A320 was analysed, whose take-off is aided by the MAGLEV technology. The value of the most important parameters and the different configurations are presented in [11] and listed in Tab. 1. Three different scenarios to operate the GABRIEL system during take-off was analysed (Tab. 2). Departure scenarios were established based on take-off scenarios of possible using the GABRIEL concept [13]. The 3D trajectory of climbing to the FL100 (3050 m) above the take-off level for the same scenarios, complying with conditions of Schiphol airport was determined. The path constraints of the problem are those that conform the aircraft's flight envelope and have been taken from the calculations and literature [2].

Tab. 1. A320-200 (WV000) configuration definitions [11]

\begin{tabular}{|l|l|l|l|}
\hline \multicolumn{1}{|c|}{ Lever position } & \multicolumn{1}{|c|}{ Flight phase } & \multicolumn{1}{c|}{ Flap angle (deg) } & Slat angle $(\mathrm{deg})$ \\
\hline 0 & Cruise & 0 & 0 \\
\hline 2 & Take-off & 15 & 22 \\
\hline 3 & Take-off/Approach & 20 & 22 \\
\hline Full & Landing & 35 & 27 \\
\hline
\end{tabular}


Tab. 2. Definition of take-off scenarios (where: CONF- see [2] and [11])

\begin{tabular}{|l|l|c|c|c|}
\hline Aircraft weight (kg) & Scenario & $\begin{array}{c}\text { Take-off } \\
\text { speed, }(\mathrm{m} / \mathrm{s})\end{array}$ & $\begin{array}{c}\text { Use of aircraft engine } \\
\text { power at lift-off }(\%)\end{array}$ & Flap \\
\hline $\begin{array}{l}71168,44 \\
\text { (aircraft } \\
\text { landing gear) }\end{array}$ & without & 75 & $\cong 0$ & CONF 2 \\
\cline { 2 - 5 } & II-Accelerated & 75 & 100 & CONF 2 \\
\hline
\end{tabular}

Tab. 3. Boundary conditions for climb path optimization

\begin{tabular}{|l|r|c|}
\hline \multicolumn{1}{|c|}{ Parameter } & Value & Unit \\
\hline$x_{0}$ & 0 & {$[\mathrm{~m}]$} \\
\hline$y_{0}$ & 0 & {$[\mathrm{~m}]$} \\
\hline$h_{0}$ & 0 & {$[\mathrm{~m}]$} \\
\hline$V_{0}=V_{L O F}($ depending on scenario) & Tab. 2. & {$[\mathrm{~m} / \mathrm{s}]$} \\
\hline$\gamma_{0}$ & 0.02 & {$[\mathrm{rad}]$} \\
\hline$h_{f}$ & FL100 & - \\
\hline$V_{f}$ & 275 & {$[\mathrm{knot}]$} \\
\hline$R O C_{f}$ & 2200 & {$[\mathrm{fpm}]$} \\
\hline
\end{tabular}

Search for optimal trajectories minimizing emission must be done in a realistic flight domain. Indeed, operational procedures are performed with respect to parameter limits related to the safety of flight and the operational modes of the aircraft. The path constraints are equal for all phases:

- Position coordinates: $x \geq 0, h \geq 0$,

- Lift coefficient: $C_{L}<C_{\text {Lmax }},\left(\alpha<\alpha_{C R}\right)$,

- Load factor: $n<1.2$,

- Thrust: $T(h, V)<T_{\max }(h, V)$.

The 3D trajectory of climbing to the FL100 (3050 m) above the take-off level for the analysed scenarios, complying with conditions of Schiphol airport was determined. It was assumed the takeoff from the runway RWY 27 with yaw angle $\psi=265$ [deg] (Fig. 1.). The orientation of established coordinate system, and location of high population areas to be avoided relative runway is also shown on Fig. 1. The 3D form of the optimal trajectory is shown on Fig. 2.

The trajectory of climbing to the cruise height of flight (Tab. 3) for different take-off scenarios (Tab. 2) was determined. The taking-off speeds followed from the analysed scripts described in [15]. The calculations were carried out minimizing the fuel consumption during the climbing phase. The values of the optimized parameters were determined by traditional methods of static optimization. The optimal change of trajectory parameters are presented on Fig. 3-10. Weight of fuel used is presented in Tab. 4.

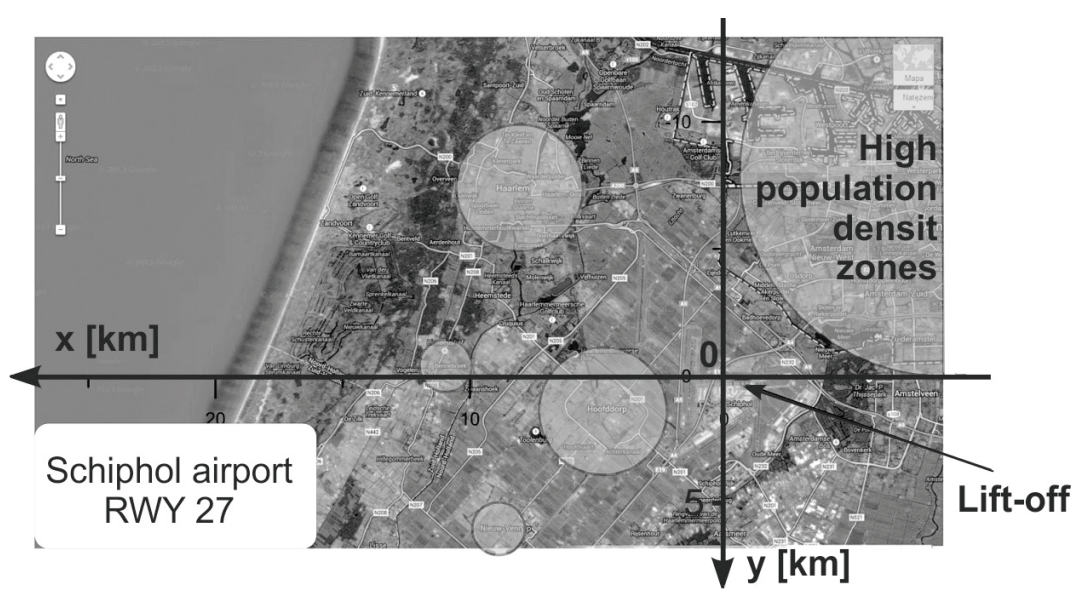

Fig. 1. Situation map with high population areas shown as prohibited area 




Fig. 2. 3D form of the optimal trajectories for three scenarios and reference aircraft

Tab. 4. Fuel weight used for climb

\begin{tabular}{|l|l|}
\hline Take-off case & Fuel weight $(\mathrm{kg})$ \\
\hline Conventional & 449.49 \\
\hline Scenario I & 418.38 \\
\hline Scenario II & 386.00 \\
\hline Scenario III & 329.35 \\
\hline
\end{tabular}

At scenario I, (accelerated) aircraft lifts-off with lift-off speed and configuration identical than reference aircraft, but it has less weight [11] and engines working at idle [13]. At this scenario, aircraft has low manoeuvrability; therefore, trajectory has the smallest curvature. Necessity of engine acceleration cause the smallest height increase (Fig. 3 and 10). Reference aircraft has engines working at full throttle but it has greater weight than modified versions. It also affect to low manoeuvrability and small curvature of the trajectory. At scenario II (conventional) aircraft lifts-off with lift-off speed and configuration identical than reference aircraft and at scenario I, but it has weight less than reference a/c [11] and engines working at full throttle [13]. Thanks to that, it has better manoeuvrability, and the trajectory can shaped otherwise. Aircraft can pass round prohibited zone (Haarlem) from the east side. The best conditions for optimal shaping of the trajectory have the aircraft at scenario III (unconventional). The biggest lift-off speed and en route configuration at whole phase (Tab. 2) brings about the better manoeuvrability. Therefore, the trajectory has largest curvature.

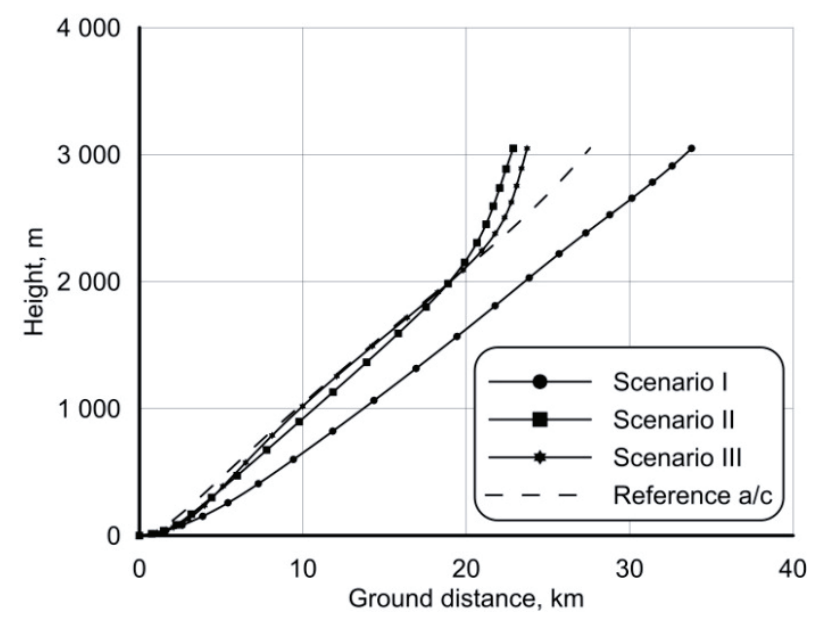

Fig. 3. The optimal climb trajectory

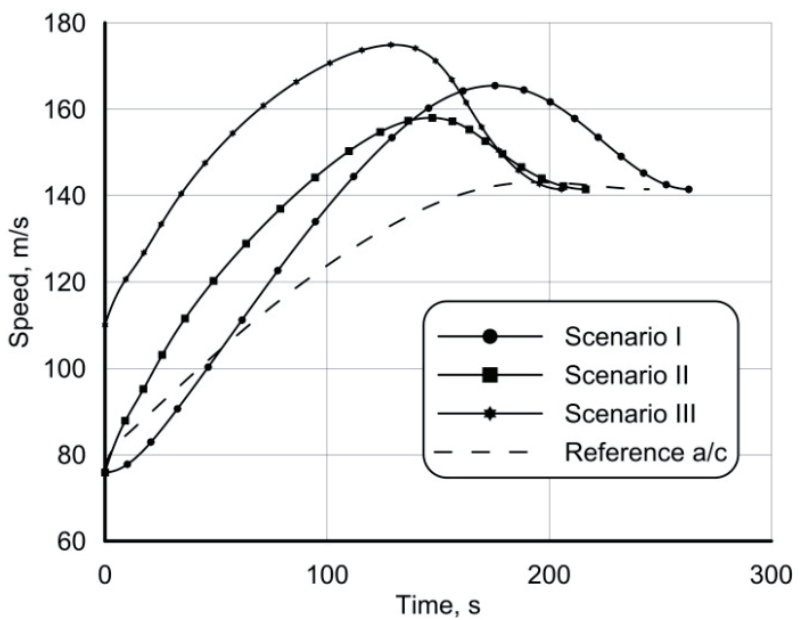

Fig. 4. The optimal change of speed 




Fig. 5. The optimal change of trajectory angle

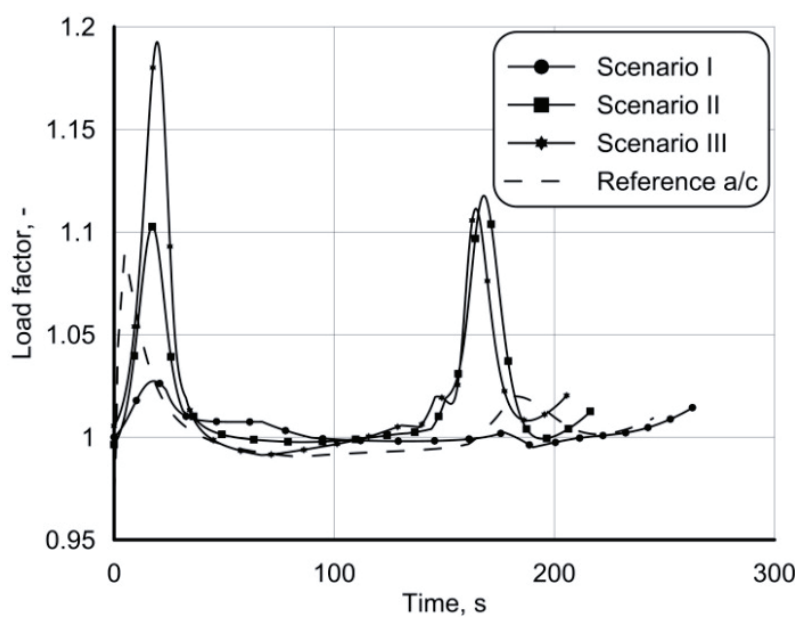

Fig. 6. The optimal change of load factor

Figure 4 presents the change of optimal speed of the aircraft during climb for three scenarios (Tab. 2) with comparison to optimal speed of reference aircraft. For scenario I, speed increases slowly due to low lift-off speed and low throttle level. Scenario II is characterized by greater speeds due to full throttle at lift-off. Speeds at first stage (to 40 [s]) are limited by lift-off configuration (flaps extended). For scenario III speed increases to the highest values. From a point of view of fuel consumption minimization, higher lift-off speed is preferred and lift-off at cruise configuration. On Fig. 5, the optimal change of trajectory angle is presented. Scenario I is characterized by small changes of trajectory angle. It is caused by small speeds. In scenario III, a little greater changes of trajectory angle come out of greater speeds, giving higher aircraft manoeuvrability. Scenario II gives the biggest trajectory angle at part in which aircraft climbs dynamically. Load factors (Fig. 6) for scenario I changes in very small range due to small speed reserve (low manoeuvrability). At scenario II and III, load factors are limited by usable boundary. Higher speeds and manoeuvrability gives possibility to shape the trajectory in a different way. Fig. 7 and 8 shows the optimal changes of yaw and roll angle. Fig. 9 shows the changes of optimal lift coefficient for three scenarios. The optimal lift coefficient for high lift-off speed (third scenario) changes in the smallest range. For scenarios I and II, the assumed flaps retraction speed is $3 \mathrm{deg} / \mathrm{s}$. Fig. 10 presents the optimal change of vertical speed (rate of climb) during initial climb stage. For scenarios II and III, maximum values of vertical speed are similar. It is caused by maximum available thrust of power unit (quasi-static climb). Maximum vertical speed for first scenario is significantly smaller than two others are but greater than currently realized by reference aircraft. It is caused by higher energy coming out of high lift-off speed utilized for dynamic vertical manoeuvre.

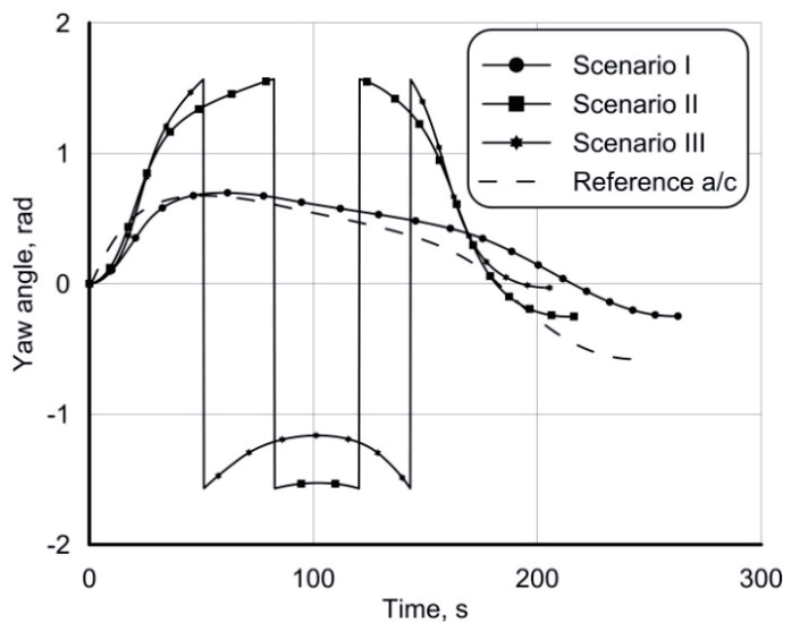

Fig. 7. The optimal change of yaw angle

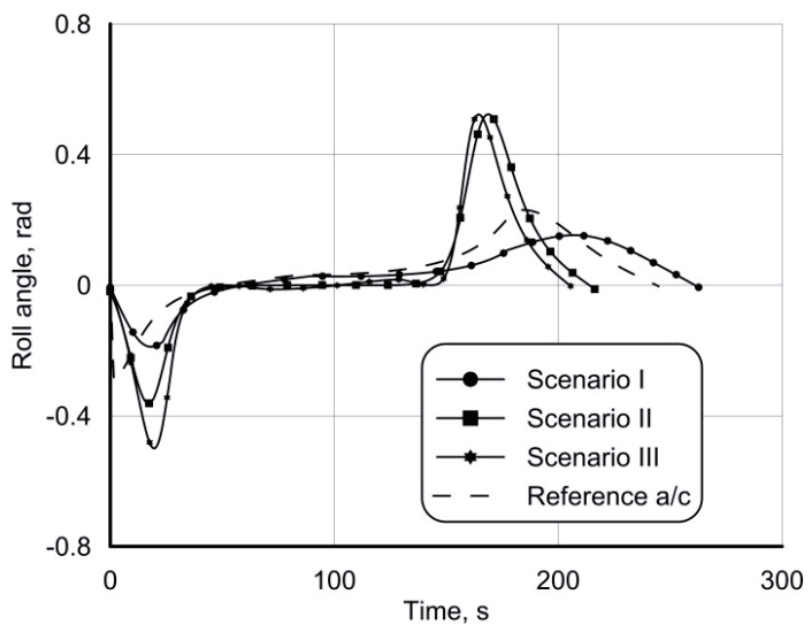

Fig. 8. The optimal change of load factor 




Fig. 9. The optimal change of lift coefficient

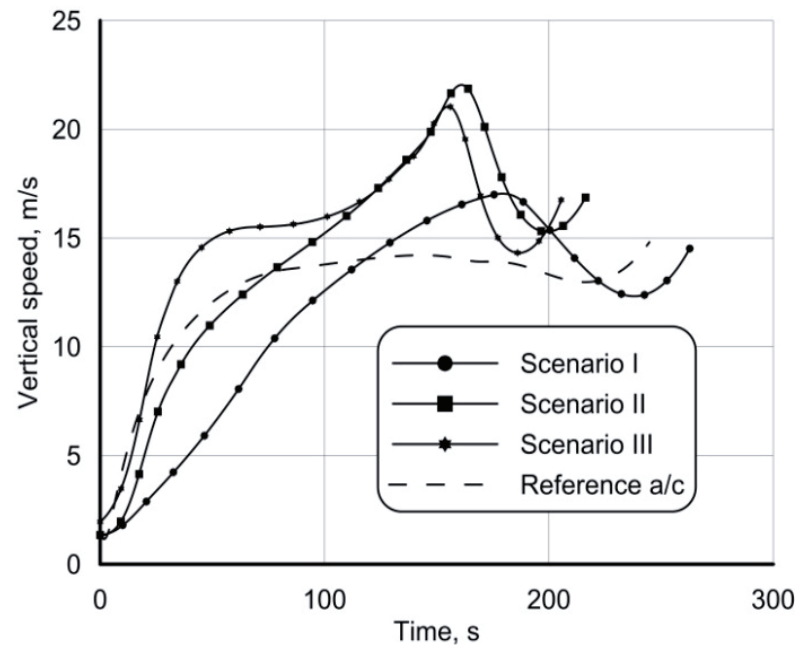

Fig. 10. The optimal change of vertical speed

\section{Conclusion}

The work concerned determining the optimal trajectory of the initial phase of the transport aircraft climb aided in the phase of acceleration by the system using the magnetic levitation phenomenon. The conditions different from a traditional take-off (higher take-off speed, lack of undercarriage, en route configuration) allows assuming that both optimal profiles of the aircraft climb should be different from currently performed. Determining the climb trajectory guaranteeing minimization of the fuel consumption would also give a solution characterized by minimal emission of substances harmful for the environment. To determine the optimal trajectory of climb the Ritz-Galerkin method was used which is the method of approximate solution of boundary value problems, which guarantees obtaining the results thorough enough for practical purposes. Further works should concern determining the optimal trajectory regarding minimization of harmful substance and noise emission, with use of more accurate calculation models.

\section{Acknowledgement}

This material is based upon the work supported by the European Commission, under Contract No 284884, "Integrated Ground and on-Board system for Support of the Aircraft Safe Take-off and Landing" - GABRIEL.

\section{References}

[1] Abdallah, L, Haddou, M., Khardi, S., Optimization of Operational aircraft parameters reducing noise emission, Applied Math. Sc., 4:1, 515-535, 2010.

[2] AIRBUS, A320 Airplane Characteristics for Airport Planning, AIRBUS S.A.S., 1985.

[3] Customer Services, Technical Data Support and Services, 31707 Blagnac Cedex, FRANCE.

[4] Bellman, R., The theory of dynamic programing, The Rand Corporation, Santa Monica, California 1954.

[5] Elbir, T., Estimation of Engine Emissions from Commercial Aircraft at a Midsized Turkish Airport, Journal of Environmental Engineering, 210-215, 2008.

[6] Etkin, B., Reid, L. D., Dynamics of Flight, John Wiley and Sons, third edition, 1996.

[7] Khan, S., Flight Trajectories Optimization, ICAS 2002 Congress, Toronto, Canada 2002.

[8] Khardi, S., Aircraft Flight Path Optimization, The Hamilton-Jacobi-Bellman Considerations, Applied Mathematical Sciences, Vol. 6, No. 25, 1221-1249, 2012.

[9] Majka, A., Klepacki, Z., Orkisz, M., Pawluczy-Majka, J., Schmollgruber, P., Sibilski, K., Felisiak, P., Wrobel, M., Aircraft weight breakdown and energy balance calculation, 
Deliverable D2.2. Integrated Ground and on-Board system for Support of the Aircraft Safe Take-off and Landing - GABRIEL, EU Project No. 24884, Rzeszow 2011.

[10] Majka, A., Take-off aided by magnetic levitation technology, Aircraft Engineering and Aerospace Technology, Emerald Group Publishing Limited, T. 85, Z. 6, pp. 435-442, 2013.

[11] Majka, A., Klepacki, Z., Orkisz, M., Pawluczy-Majka, J., Wygonik, P., Sibilski, K., Felisiak, P., Wrobel, M., Rohacs, D., Rohacs, J., Effect of maglev on aircraft characteristics geometrics, weight, aerodynamics, flight performance, Deliverable D2.11, Integrated Ground and on-Board system for Support of the Aircraft Safe Take-off and Landing - GABRIEL, EU Project No. 284884, Rzeszow 2013.

[12] Pontryagin, L., Boltyansky, V., Gamkrelidze, V, Mischenko, E., Mathematical Theory of Optimal Processes, Wiley-Interscience, New York 1962.

[13] Pool, D., Voskuijl, M., Falkena, W., Siepenkötter, N., Majka, A., Falkowski, K., Wróblewski, W., Sibilski, K., Simulation Technology and Simulation of the GABRIEL Concept, Deliverable D4.2, Integrated Ground and on-Board system for Support of the Aircraft Safe Take-off and Landing - GABRIEL, EU Project No. 284884, Delft, 2013.

[14] Prats, X., Puig, V., Quevedo, J., Nejjari, F., Optimal departure aircraft trajectories minimising population annoyance, 3rd International Conference on Research in Air Transportation (ICRAT), Fairfax, Virginia, USA 2008.

[15] Prats, X., Quevedo, J., Puig, V, Trajectory Management for Aircraft Noise Mitigation, ENRI International Workshop on ATM/CNS, Tokyo, Japan 2009.

[16] Rohacs, J., Rohacs, D., Schmollgruber, P., Voskuijl, M., GABRIEL operational concept, Deliverable D2.9, Integrated Ground and on-Board system for Support of the Aircraft Safe Take-off and Landing - GABRIEL, EU Project No. 284884, Budapest 2012.

[17] Taranienko, W. T., Momdzi, W. G., Simple variational method in boundary value problems of flight dynamics, Maszinostrojenije, Moscow 1986.

[18] Wijnen, R. A. A., Visser, H. G., Optimal departure trajectories with respect to sleep disturbance, 7th AIAA/CEAS Aeroacoustics Conference, Maastricht, The Netherlands 2001. 